OPEN ACCESS

Edited by:

Jianming $L i$,

Soochow University, China

Reviewed by:

Jun Zhou,

Southern Medical University, China

Tong Shen,

Soochow University, China

Su Yao,

Guangdong Provincial

People's Hospital,

China

*Correspondence:

Guo-Hui Fu

fuguhu@263.net

Li-Dong Zu

zulidong@163.com

Specialty section:

This article was submitted to Gastrointestinal Cancers,

a section of the journal

Frontiers in Oncology

Received: 07 January 2021 Accepted: 17 March 2021

Published: 21 April 2021

Citation:

Yu Y, Wang J-L, Meng L-L, Hu C-T, Yan Z-W, He Z-P, Shi X-Q, Fu G-H and

Zu L-D (2021) DDX54 Plays a

Cancerous Role Through Activating

P65 and AKT Signaling Pathway in

Colorectal Cancer.

Front. Oncol. 11:650360.

doi: 10.3389/fonc.2021.650360

\section{DDX54 Plays a Cancerous Role Through Activating P65 and AKT Signaling Pathway in Colorectal Cancer}

\author{
Yi Yu ${ }^{1}$, Jing-Long Wang ${ }^{2}$, Li-Li Meng ${ }^{2}$, Chun-Ting $\mathrm{Hu}^{2}$, Zhao-Wen Yan ${ }^{2}$, Zhi-Ping $\mathrm{He}^{2}$, \\ Xiao-Qin Shi ${ }^{1}$, Guo-Hui Fu ${ }^{1,2^{*}}$ and Li-Dong $\mathrm{Zu}^{{ }^{2 *}}$ \\ ${ }^{1}$ Pathology Center, Shanghai General Hospital, Shanghai Jiao Tong University, Shanghai, China, ${ }^{2}$ Department of Pathology, \\ Shanghai Jiao Tong University School of Medicine, Shanghai, China
}

Colorectal cancer (CRC) is one of the most malignant cancers, and its incidence is still steadily increasing. The DDX RNA helicase family members have been found to play a role in various cancers; however, the role of DDX54 in colorectal cancer is still unclear and needed to be defined. Here, we found DDX54 was overexpressed in CRC tissues by the label-free mass spectrum, which was also verified in tissue microarray of colon cancer, as well as the CRC cell lines and TCGA database. High DDX54 level was correlated with tumor stage and distant metastasis, which always indicated a poor prognosis to the CRC patients. DDX54 could promote the proliferation and mobility of CRC cells through increasing the phosphorylation level p65 and AKT leading to the tumorigenesis. Here, we have preliminarily studied the function of DDX54 in CRC, which would improve our understanding of the underlying biology of $\mathrm{CRC}$ and provide the new insight that could be translated into novel therapeutic approaches.

Keywords: DDX54, p65, AKT, proteomics, colorectal cancer

\section{INTRODUCTION}

Colorectal cancer (CRC) is a public health problem and is a major cause of morbidity and mortality worldwide as the third most common cancer and the second leading cause of cancer death (1). Although surgical resection is a relatively curative method for the treatment of colon cancer, metastasis and recurrence often occur after surgery, and the prognosis remains very poor $(2,3)$. Although extensive studies have demonstrated that CRC is a multi-factorial disease and frequently associated with physical, chemical, biological and genetic factors (4), and emerging evidence indicates that CRC is also a heterogeneous disease, arising through accumulation of genetic and epigenetic alterations, the etiology of CRC remains to be fully elucidated. Thus, it is important to identify the genes that contribute to CRC development.

DEAD-box proteins belong to the superfamily 2 (SF2) helicases (5). DEAD-box proteins are ATP dependent RNA helicases and include 38 members in humans (5). DEAD-box proteins function throughout the lifetime of cellular RNAs from synthesis to biological activity, and to inevitable decay (5). Recently, DEAD RNA helicase families were found to be expressed widely in the cancers including DDX5 in breast cancer (6) and lung cancer (7), DDX3 in prostate cancer (8) 
and hepatocellular cancer (9), and DDX27 in CRC (10) and gastric cancer (11). They are dysregulated in cancer in the form of involvement in chromosomal translocation, down-regulation, and over-expression of cancerous genes. DDX54, a member of the DEAD-box RNA helicase protein family, has been identified as a hormone-dependent interacting protein of estrogen receptors (ERs) (12) and CAR-binding protein (13). In addition, DDX54 also had a role on DNA damage repair (14) and myelination in oligodendrocytes (15). Recently, it was reported that the association of DDX54 to SNHG10 and PBX3 led to co-stability of each other and the complex could facilitate cell growth and motility in gastric cancer (16).

The important role of nuclear factor- $\mathrm{KB}$ (NF- $\mathrm{KB}$ ) has been widely confirmed in various kinds of cancer (17). In colorectal cancer, NF- $\mathrm{KB}$ has a correlation with cancer-related processes involving the cell proliferation, apoptosis, angiogenesis, and metastasis (18). Targeting NF- $\kappa B$ pathway components may be a preventive measures and effective treatment approach to improve the patients' overall outcome and quality of life. NF$\kappa \mathrm{B}$ is a heterodimer protein that consists of two subunits, p65 (RelA) and p50 which are required for activation and nuclear translocation of NF- $\mathrm{KB}$. The promotive role of NF- $\mathrm{KB}$ in mediating colon carcinogenesis is illustrated by its regulation of a great diversity of genes positioned at the interface between cell proliferation, survival, and evasion from apoptosis. Mounting evidences support the role of NF- $\mathrm{KB}$ in regulating the expression of anti-apoptotic members of the Bcl-2 family like $\mathrm{Bcl}-2$ and Bcl-xL (19). As the members of the IAP gene family, $\mathrm{XIAP}$ and Survivin have been identified as downstream targets of NF- $\mathrm{\kappa B}$ signaling pathway (20). Regulation of NF- $\mathrm{\kappa B}$ activity occurs at multiple levels. Hence, it is not simply adequate to understand the mechanism of nuclear localization and DNA binding of NF- $\mathrm{KB}$ subunits. Comprehending the molecular mechanism behind the regulation of each subunit of NF- $\mathrm{KB}$ complex and determination of their functionality will help to elucidate the NF- $\mathrm{KB}$ pathway as a whole.

The PI3K/AKT/mTOR pathway is another key regulator point of intracellular metabolism, cell growth and survival, angiogenesis, and invasiveness, which is involved in carcinogenesis and progression (21). This signaling pathway was verified to response to various intracellular and extracellular stimuli, such as inflammatory cytokines, growth factors and carcinogenic agents (22). AKT pathway is activated in a majority of human cancers due to mutations in PIK3CA or inactivation and decreased function of PTEN $(23,24)$.

In this study, we had screened the proteins with different expression level through proteomic analysis to found out the potential molecular marker. We found DDX54 was highly expressed in CRC tumor tissues compared to normal tissues. We also observed that CRC patients always had a relatively longer survival time with low DDX54 expression than those with high expression by colon cancer tissue microarray and prognosis prediction with online database. Moreover, DDX54 indeed inhibited the proliferation and metastasis in CRC cells. DDX54 might be a new molecular marker for CRC prognosis prediction.

\section{MATERIALS AND METHODS}

\section{Human Tissue Samples}

Human colon cancer tissue samples and para-tumor tissue samples were obtained from the pathology center, Shanghai General Hospital/Faculty of Basic Medicine, Shanghai Jiao Tong University School of Medicine. These samples were immediately frozen in tubes and stored in liquid nitrogen after surgical resection. Written informed consent was obtained from each patient. Human CRC tissue samples for tissue microarray of 96 patients and corresponding adjacent specimens were obtained from patients who underwent colectomy or rectectomy at the Shanghai General Hospital (Shanghai, China) from October 2010 to August 2013. None of the patients were treated with immunotherapy, radiotherapy, or chemotherapy before surgery. The study was approved by the ethics committees of Shanghai Jiao Tong University, School of Medicine (Shanghai, China).

\section{Label-Free Mass Spectrometry Analysis}

Sample was ground individually in liquid nitrogen and lysed with lysis buffer which containing $100 \mathrm{mM} \mathrm{NH} 4 \mathrm{HCO} 3(\mathrm{pH}$ 8), $6 \mathrm{M}$ urea and $0.2 \%$ SDS, followed by $5 \mathrm{~min}$ of ultrasonication on ice. The lysate was centrifuged at $12,000 \mathrm{~g}$ for $15 \mathrm{~min}$ at $4^{\circ} \mathrm{C}$, and the supernatant was transferred to a clean tube. Extracts from each sample were reduced with $10 \mathrm{mM} \mathrm{DTT}$ for $1 \mathrm{~h}$ at $56^{\circ} \mathrm{C}$, and subsequently alkylated with sufficient iodoacetamide for $1 \mathrm{~h}$ at room temperature in the dark. Then samples were completely mixed with four times volume of precooled acetone by vortexing and incubated at $-20^{\circ} \mathrm{C}$ for at least $2 \mathrm{~h}$. Samples were then centrifuged and the precipitation was collected. After washing twice with cold acetone, the pellet was dissolved by dissolution buffer, which contain $0.1 \mathrm{M}$ triethylammonium bicarbonate (TEAB, $\mathrm{pH} 8.5$ ) and $6 \mathrm{M}$ urea. The LC-MS/MS spectra were collected and subjected to comparison with the UniProt human proteome database using Proteome Discoverer 1.4 (Thermo Fisher). Proteins were included for analysis when represented by at least two unique peptides. The raw MS data were processed using the MaxQuant software (version 1.5.3.8) (25) and matched to peptide sequences in the human UniProt protein database by the Andromeda algorithm (26). Relative, label-free quantification of the proteins was done using the MaxLFQ algorithm (27). The protein group files were imported into Perseus software (version 1.5.2.4) (28) to perform statistical analysis and validation. Hierarchical clustering was performed after $\mathrm{z}$-score normalization of the data within Euclidean distance. Gene ontology (GO) analysis and KEGG analysis of differentially expressed proteins were generated by DAVID 6.8 (http://https:// david.ncifcrf.gov/).

\section{Xenograft GC Nude Mice Model}

Female athymic BALB/c nude mice (6-8 weeks old) were purchased from Shanghai Experimental Animal Center, Chinese Academy of Science. $5 \times 10^{6}$ RKO cells infected with virus of siRNAs control and siRNAs targeted DDX54, were suspended in PBS and then subcutaneously injected into the nude mice. After two weeks, the tumors reached $\sim 200 \mathrm{~mm}^{3}$. 
All mice were sacrificed after anesthesia and tumor size and weight were measured. Tumor volume was calculated, $\mathrm{V}=$ lengthwidth $2 / 2 \mathrm{~mm}^{3}$. The experiments were approved by the animal research committee in Shanghai Jiao Tong University.

\section{Cell Culture}

Human colorectal cancer cell lines were purchased from the Cell Bank of the Shanghai Institute for Biological Science (Shanghai, China). RKO, Caco2, SW620. SW480, HCT116, and DLD-1 were cultured in RPMI-1640 or high-glucose DMEM (Hyclone, Thermo Fisher, USA) medium supplemented with $10 \%$ fetal bovine serum (Hyclone) and $1 \%$ penicillin/streptomycin (Invitrogen, Carlsbad, CA, USA) at $37^{\circ} \mathrm{C}$ in a humidified atmosphere with $5 \% \mathrm{CO}_{2}$. The other cells were cultured in DMEM with $10 \%$ FBS.

\section{Stable Cancer Cell Construction}

DDX54 and shDDX54 virus stock solutions were purchased from Sangon Biotech (Shanghai, China). A day before virus infection, the colon cells were seeded into the six-well plate and grew well. The virus stock was diluted according to MOI (1:10 for v-DDX54 and 1:20 for v-siDDX54) and dropped into the wells along with polybrene addition. $24 \mathrm{~h}$ later, the diluted virus infected the cell again. Then the selective medium with antibiotics was placed. And after 2 weeks, the cells were cultured for assay.

\section{Quantitative Real-Time PCR (qRT-PCR)}

Total RNA was extracted from cells or tissue samples after homogenization using Trizol reagent (15596-026, Invitrogen) according to the manufacturer's instructions, and were reverse transcribed (RD0037A, Takara) into cDNA with specific RT primers. The relative mRNA levels were analyzed by qRT-PCR using a one-step SYBR PrimeScript ${ }^{\mathrm{TM}}$ RT-PCR Kit II (RR420A, Takara) in an ABI 7500 fast fluorescence temperature cycler. Glyceraldehyde 3-phosphate dehydrogenase (GAPDH) was chosen as the control for DDX54 normalization. The relative expression level of DDX54 was calculated using the $2^{-\Delta \Delta \mathrm{Ct}}$ method after normalization. All experiments were repeated three times. The primer sequences were:

$\begin{array}{ll}\text { GAPDH-F } & \text { CTCCTCCTGTTCGACAGTCAGC } \\ \text { GAPDH-R } & \text { CCCAATACGACCAAATCCGTT } \\ \text { DDX54-F } & \text { TATGACAACAGCCTCAAGAT } \\ \text { DDX54-R } & \text { AGTCCTTCCACGATACCA }\end{array}$

\section{Western Blotting}

The whole cells were washed and lysed in RIPA buffer (89900, Thermo Scientific) supplemented with PMSF (phenylmethylsulfonyl fluoride) and protease inhibitors. Protein concentrations were determined with a BCA assay kit (Pierce, Rockford, IL, USA). Then the lysates were mixed with loading buffer, analyzed by $10 \%$ sodium dodecyl sulfate-polyacrylamide gel electrophoresis and then transferred onto polyvinylidene difluoride membranes (Millipore, Billerica, MA). After blocked with 5\% skim milk in TBST at room temperature for $1 \mathrm{~h}$, the membranes were incubated with different primary antibodies, including anti-DDX54 (1:1000, Proteintech, China), anti-P65, anti-p-P65, anti-AKT, antip-AKT, anti-mTOR, anti-p-mTOR, (1:1000, Cell Signaling
Technology, Danvers, MA, USA), and anti-GAPDH (1:5000, Yeason, China) in $5 \%$ milk/TBST buffer at $4^{\circ} \mathrm{C}$ overnight, and then probed with horseradish peroxidase-conjugated anti-mouse or anti-rabbit IgG (1:5000, Jackson Immunoresearch Laboratories, West Grove, PA, USA) for $1 \mathrm{~h}$. After washing three times with TBST, the membrane was developed with enhanced chemiluminescent plus substrate (Merck Millipore, Billerica, MA, USA), and the signal was recorded by Fluorchem E System (ProteinSimple, Santa Clara, CA, USA).

\section{In Vitro Cell Proliferation Assay}

The cell proliferation assay was performed using a CCK-8 (Cell Counting Kit-8) kit (Dojindo, Japan). Colon cancer cells including RKO, Caco2, SW620 and HCT116 were seeded at a density of $2.5 \times 10^{3}$ cells per well into 96-well plates with each well containing $200 \mu \mathrm{l}$ medium. After culture for $24 \mathrm{~h}$, the OD values were measured at $450 \mathrm{~nm}$. The inhibitory agents' treatments were carried every day $24 \mathrm{~h}$ after the cell seeding.

\section{Immunohistochemistry}

Tumor specimens were fixed in $10 \%$ formalin overnight and embedded in paraffin. To observe DDX54 expression in CRC tissues, deparaffinized slides were treated with $3 \% \mathrm{H}_{2} \mathrm{O}_{2}$ and subjected to antigen retrieval using $0.01 \mathrm{M}$ citric buffer solution ( $\mathrm{pH}$ 6.0). After overnight incubation with the rabbit anti-DDX54 antibody (1:100 dilution, Abnova) at $4^{\circ} \mathrm{C}$, the slides were incubated for $15 \mathrm{~min}$ at room temperature with horseradish peroxidase-labeled polymer conjugated to a secondary antibody (Max Vision ${ }^{\mathrm{TM}} \mathrm{Kit}$ ) and incubated with diaminobenizidine (DAB) for $2 \mathrm{~min}$. The slides were then counterstained with hematoxylin. Appropriate positive and negative controls were tested in parallel. The IHC staining results were assessed by two pathologists without prior knowledge of patient data. The percentage of stained cells was scored as follows: $0 \%$ as $0 ; 1 \%$ to $10 \%$ as $1 ; 10 \%$ to $50 \%$ as $2 ; 51 \%$ to $80 \%$ as $3 ; 81 \%$ to $100 \%$ as 4 , and the intensity of staining: no staining as 0 ; weak staining as 1 ; moderate staining as 2 ; strong staining as 3 . Then multiplying the two was the score of each tissue (score range, $0-12$ ) and the cutoff value was 6 to distinguish the high and low DDX54 staining.

\section{Statistical Analysis}

Data are expressed as the mean \pm standard deviation (SD). Student's t-test was performed for continuous variables. The correlations of DDX54 staining and clinicopathological features were analyzed using the Pearson $\chi^{2}$ test. The survival analysis was carried out by the Kaplan-Meier method and evaluated using the log-rank test. The Cox proportional hazards regression model was used for univariate and multivariate analysis to evaluate the hazard ratio (HR) of prognostic factors. $\mathrm{P}<0.05$ was considered significant.

\section{RESULTS}

\section{MS Analysis in Colon Cancer}

In order to explore the mechanism which led to the tumorigenesis in colorectal cancer, we enrolled six pairs CRC 
patients and collected the tumor tissues and the adjacent normal tissues. The different protein expressions were analyzed between the tumor tissues and adjacent normal tissues through the mass spectrum (MS) technology. A total of 6847 proteins were identified from all tissue samples and 537 proteins were found to be differentially expressed $(p<0.05)$ (Figure 1A). 170 proteins were up-regulated and 163 proteins were down-regulated which displayed 1.5-fold quantitative alterations. According to the data from GO (gene ontology) database and KEGG (Kyoto encyclopedia of genes and genome) pathway database, 537 proteins were analyzed (Figures 1B, C). KEGG analyses showed these proteins were most involved in metabolic pathways, focal adhesion, proteoglycans in cancer, and regulation of actin cytoskeleton, etc.

\section{Verification of MS in Colon Cells and Colon Cancer Patients}

In order to discover the differentially expressed proteins in colorectal cancer tissues compared to the adjacent benign tissues, four criterions were followed: 1) the gene should be highly expressed in the cancer tissues, 2) the up-regulated trend should be consistent in all patients, 3 ) the carcinogenic role of its similar proteins should be reported previously, 4) the result or the overexpressed gene should be verified in followed samples of the CRC patients and cell lines. Eventually, DDX54 was picked out to be investigated in our study. To confirm the alterations of protein expression revealed by quantitative proteomic analyses, DDX54 was determined by WB and qRT-PCR. The results were consistent with the quantitative proteomic analyses (Figures 2A, B). The DDX54 expression was also detected in colorectal cancer cell lines and the results showed that DDX54 was significantly overexpressed in colon cancer cell lines compared to $t$ he normal cell line NCM-460 (Figures 2C, D).

Moreover, to further determine the expression status of DDX54 in CRC patients, immunohistochemistry staining was performed with tissue microarray containing 96 colon cancer tissues and 66 normal tissues (Figure 2E). And the staining score was calculated and summarized in Figure 2F. The correlation of DDX54 expression with clinicopathological characteristics of CRC patients was analyzed, the results determined by Chi-
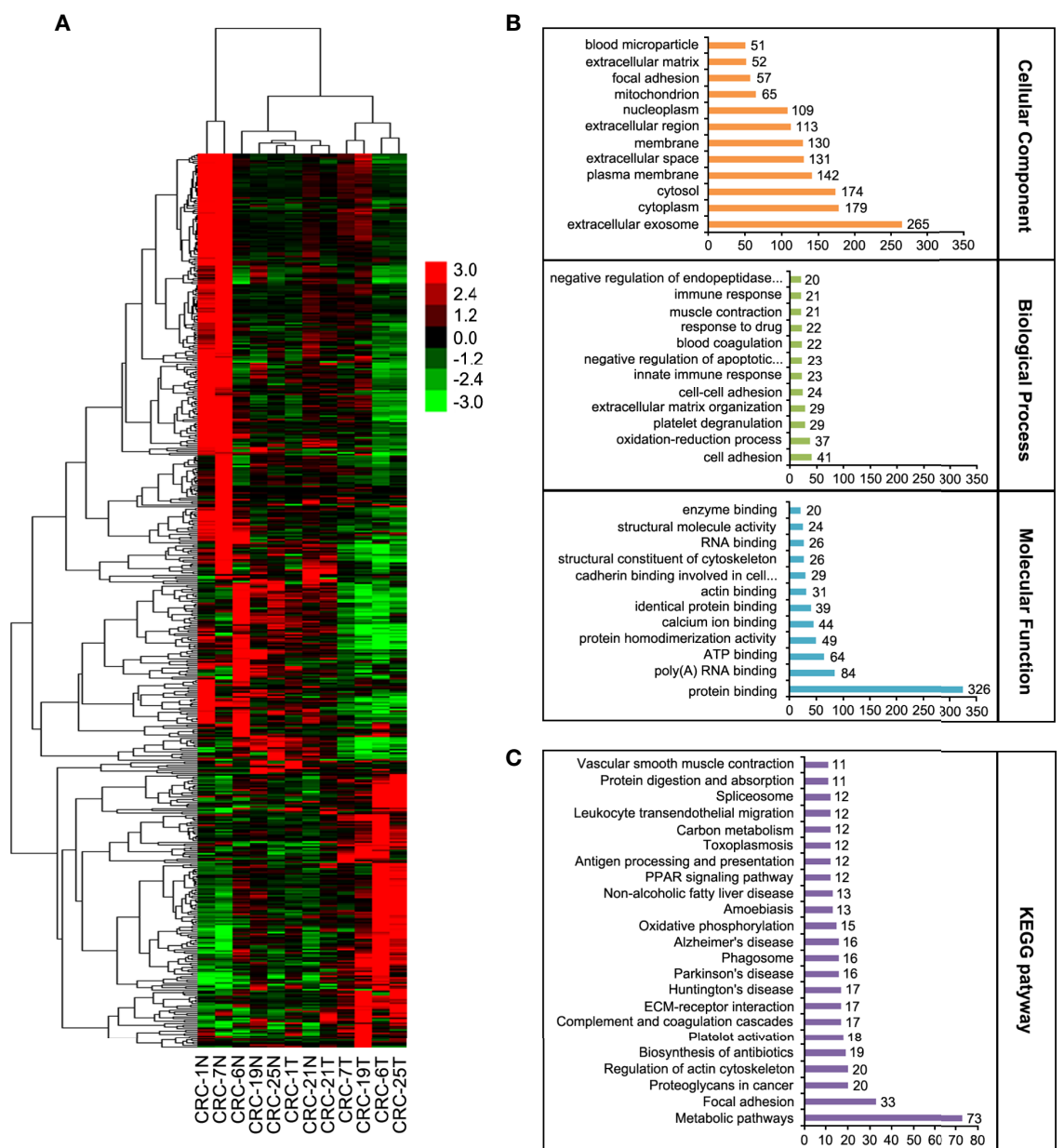

C

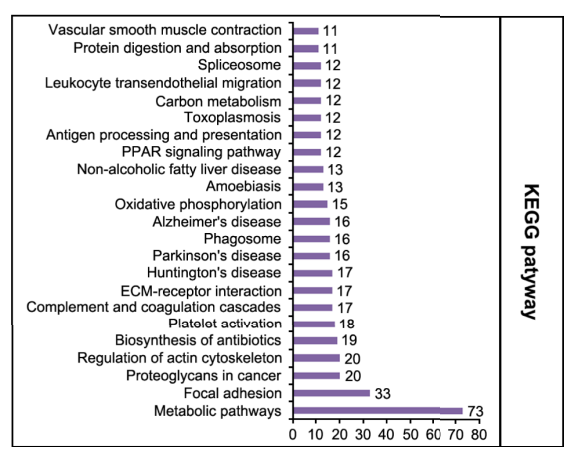

FIGURE 1 | Label-free quantitative proteomic analyses. (A) The heat map of LC-MS/MS data analysis from the six CRC tumor tissues and their adjacent normal tissues. (B) Classification of differential proteins according to the GO analyses. (C) Classification of differential proteins according to the KEGG analyses. 
square test were shown in Table 1. High DDX54 expression was significantly associated with tumor stage and distant metastasis. There was no significant relation between DDX54 expression and other clinical parameters. Univariate and Multivariate Cox regression analysis both indicated that DDX54 expression was associated with the CRC distant metastasis and revealed that high DDX54 expression was an independent and favorable prognostic indicator for OS (Tables 2, 3).
This result was further confirmed at the online websites involving the TCGA database. DDX54 was highly expressed in CRC patients than normal patients in Ualcan and GEPIA website (Figures 2G, H) at mRNA level. In human protein atlas, as the same as the mRNA level, DDX54 protein level was also upregulated in CRC patients in spite of the stage (Figure 2I). Taken together, DDX54 was indeed up-regulated in CRC cells and might have an ontogenetic role in CRC.
A

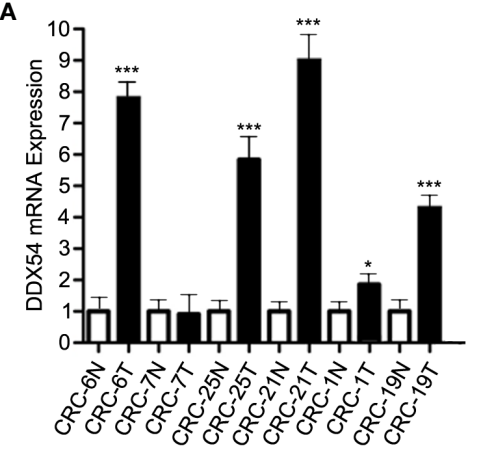

D

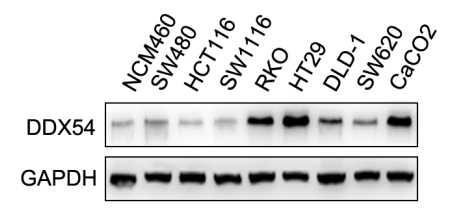

G

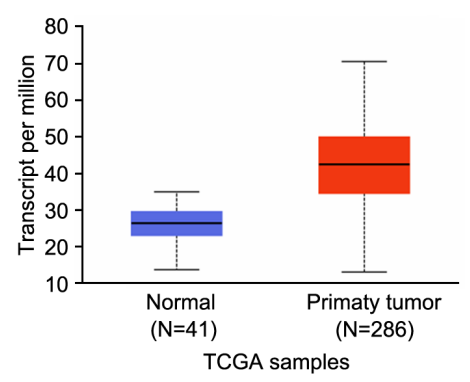

B

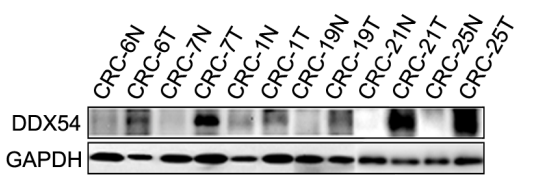

E

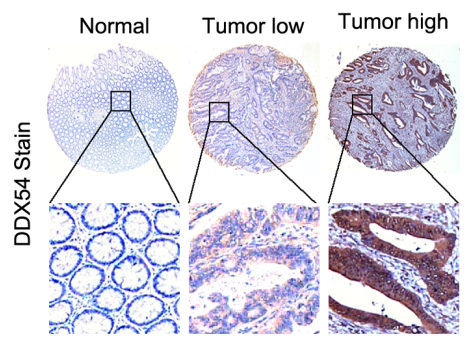

H

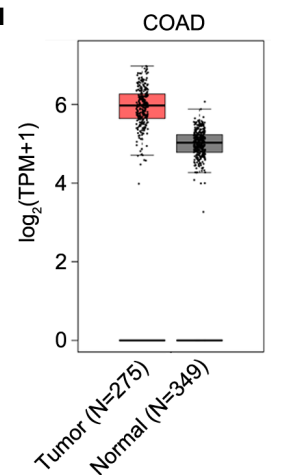

C

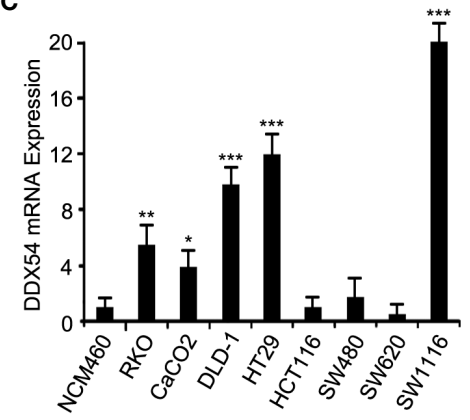

F

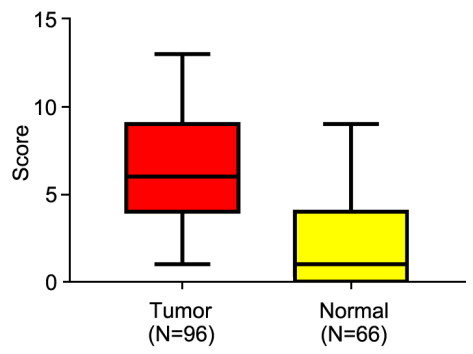

I

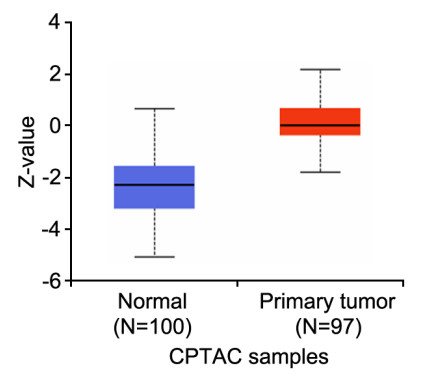

FIGURE 2 | Validation of DDX54 from different expressive proteins. (A) Q-RT-PCR was performed to evaluate the level of DDX54 in CRC patients. GAPDH was used as a normalization control. The fold change was calculated using the $2^{-\Delta \Lambda C t}$ method. ${ }^{\star}, p<0.05 ;{ }^{* \star \star}, p<0.001$. All experiments were repeated three times. (B) DDX54 expression levels in CRC patients were examined through WB and GAPDH was loaded as the control. (C) Q-RT-PCR was carried out to evaluate the level of DDX54 in CRC cell lines. GAPDH was used as a normalization control. The fold change was calculated using the $2^{-\Delta \Delta C t}$ method. ${ }^{*}, p<0.05 ;{ }^{* *}, p<0.01$; ${ }^{* \star *}, p<0.001$. All experiments were repeated three times. (D) DDX54 protein levels were determined in CRC cell lines through WB and GAPDH was selected as the control. (E) The representative images of DDX54 staining by IHC. (F) The histogram of distribution for DDX54 staining score in CRC tissues and adjacent normal tissues. (G) DDX54 mRNA expression level was analyzed in colon adenocarcinoma from TCGA database at UALCAN website (http://ualcan.path.uab.edu/). (H) DDX54 mRNA expression level was analyzed in COAD (colon adenocarcinomas) from TCGA database at GEPIA website (http://gepia.cancer-pku.cn/). (I) DDX54 protein expression was analyzed in CRC patients of CPTAC (Clinical Proteomic Tumor Analysis Consortium) database. Z-values represent standard deviations from the median across samples for the given cancer type. Log2 Spectral count ratio values from CPTAC were first normalized within each sample profile, and then normalized across samples. (http://ualcan.path.uab.edu/). 
TABLE 1 | Association between DDX54 expression and clinicopathological features of patients with colorectal cancer.

\begin{tabular}{|c|c|c|c|c|c|}
\hline \multirow[t]{2}{*}{ Clinicopathological parameters } & \multirow[t]{2}{*}{ Numbers of cases } & \multicolumn{2}{|c|}{ DDX54 expression } & \multirow[t]{2}{*}{$\chi^{2}$} & \multirow[t]{2}{*}{$P$ value } \\
\hline & & Low & High & & \\
\hline ALL & 96 & 47 & 49 & & \\
\hline Age (years) & & & & 0.042 & 0.838 \\
\hline$\geq 65$ & 48 & 23 & 25 & & \\
\hline$<65$ & 48 & 24 & 24 & & \\
\hline Gender & & & & 0.058 & 0.809 \\
\hline Male & 56 & 28 & 28 & & \\
\hline Female & 40 & 19 & 21 & & \\
\hline Tumor size, cm & & & & 1.145 & 0.308 \\
\hline$\geq 5$ & 56 & 30 & 26 & & \\
\hline$<5$ & 40 & 17 & 23 & & \\
\hline T stage & & & & 2.851 & 0.415 \\
\hline T1 & 2 & 1 & 1 & & \\
\hline T2 & 30 & 15 & 15 & & \\
\hline T3 & 50 & 27 & 3 & & \\
\hline T4 & 14 & 4 & 10 & & \\
\hline Lymph node metastasis & & & & 2.52 & 0.284 \\
\hline NO & 49 & 25 & 24 & & \\
\hline N1 & 31 & 17 & 14 & & \\
\hline N2 & 16 & 5 & 11 & & \\
\hline Distant metastasis & & & & 6.139 & $0.013^{\star}$ \\
\hline MO & 90 & 47 & 43 & & \\
\hline M1 & 6 & 0 & 6 & & \\
\hline AJCC stage & & & & 8.015 & $0.044^{\star}$ \\
\hline I & 28 & 13 & 15 & & \\
\hline$\|$ & 18 & 12 & 16 & & \\
\hline III & 44 & 22 & 22 & & \\
\hline IV & 6 & 0 & 6 & & \\
\hline Histological grade & & & & 1.309 & 0.253 \\
\hline $\mathrm{H} 1-\mathrm{H} 3$ & 78 & 33 & 45 & & \\
\hline $\mathrm{H} 4-\mathrm{H} 5$ & 18 & 14 & 14 & & \\
\hline
\end{tabular}

*Statistically significant $(P<0.05)$.

\section{High DDX54 Level Indicated a Poor Prognosis}

To evaluate the association between the DDX54 expression and prognosis of CRC, Kaplan-Meier survival curves were made and analyzed. DDX54 expression was significantly associated with the overall survival time by staining score of tissue microarray (Figure 3A). Moreover, this association between DDX54 expression and survival was also performed in CRC patients with 5 years follow-up time from TCGA database (Figure 3B). The further confirm was also obtained from the human protein atlas website (Figure 3C). These results indicated that the CRC patients with high DDX54 expression had a greatly poorer overall survival time than those with low DDX54 expression. In consideration of the important role of DDX54 on CRC, the nude mice were subcutaneously injected with RKO cells with vshDDX54 infection. The subcutaneous tumors with different volume were shown in Figure 3D, which showed that DDX54 knockdown significantly inhibited the growth of CRC cells. The tumor growth curve was calculated at different time (Figure 3E) and the tumor weight was also determined in Figure 3F.

\section{The Function of DDX54 Was Determined in CRC Cells}

In order to evaluate the function of DDX54 in CRC, we constructed the stable RKO cell line with DDX54 overexpression or DDX54 knockdown, respectively. Then the proliferative ability of these RKO cells was determined through CCK8 assay. The results showed that the RKO cells with DDX54 overexpression grew faster than those with normal DDX54. Conversely, the RKO cells with DDX54 knockdown grew slower than those normal cells (Figure 4A). The similar results of DDX54 on the proliferation were further verified in SW620 (Figure 4B) and Caco2 (Figure 4C). The ability of tumor metastasis was further determined by the Transwell experiments and the results showed that DDX54 promoted the mobility of HCT116 and SW620 cells (Figure 4D). Conversely, DDX54 knockdown inhibited the migration and invasion ability in RKO and Caco2 cells (Figure 4E). EMT was considered as a major reason for tumor mobility which was characterized by down-regulation of epithelial markers (e.g., E-cadherin) and upregulation of mesenchymal markers (e.g., Vimentin and Ncadherin) (29). In this respect, we found that E-cadherin was decreased and $\mathrm{N}$-cadherin and Vimentin were up-regulated by enhanced DDX54, then the opposite effect was observed by knockdown DDX54 (Figure 4F). E-cadherin expression was markedly increased, and $\mathrm{N}$-canherin and Vimentin expression were greatly reduced in RKO cells with DDX54 knockdown compared with the control by IHC (Supplementary Figure 1), suggesting that DDX54 promoted CRC metastasis is potentially responsible for EMT process. Moreover, the effect of DDX54 on 
TABLE 2 | Univariate Cox regression analysis of potential prognostic factors for colorectal cancer patients.

\begin{tabular}{|c|c|c|c|c|}
\hline Characteristics & $\mathbf{n}$ & HR & $95 \% \mathrm{Cl}$ & $\mathbf{P}$ \\
\hline \multicolumn{5}{|l|}{ Age (years) } \\
\hline$\geq 65$ & 48 & & & \\
\hline$<65$ & 48 & 1.761 & $0.973-3.187$ & 0.061 \\
\hline \multicolumn{5}{|l|}{ Gender } \\
\hline Male & 56 & & & \\
\hline Female & 40 & 0.717 & $0.399-1.289$ & 0.267 \\
\hline \multicolumn{5}{|l|}{ Tumor size, cm } \\
\hline$\geq 5$ & 56 & & & \\
\hline$<5$ & 40 & 0.650 & $0.362-1.167$ & 0.149 \\
\hline \multicolumn{5}{|l|}{ T stage } \\
\hline $\mathrm{T} 1+\mathrm{T} 2$ & 32 & & & \\
\hline $\mathrm{T} 3+\mathrm{T} 4$ & 64 & 2.228 & $1.102-4.503$ & $0.026^{\star}$ \\
\hline \multicolumn{5}{|c|}{ Lymph node metastasis } \\
\hline NO & 49 & & & \\
\hline $\mathrm{N} 1+\mathrm{N} 2$ & 47 & 1.535 & $0.852-2.765$ & 0.154 \\
\hline \multicolumn{5}{|l|}{ Distant metastasis } \\
\hline MO & 90 & & & \\
\hline M1 & 6 & 15.537 & $5.67-42.576$ & $<0.001^{\star \star \star}$ \\
\hline \multicolumn{5}{|l|}{ AJCC stage } \\
\hline I, ॥ & 46 & & & \\
\hline III, IV & 50 & 1.963 & $1.073-3.591$ & 0.029 \\
\hline \multicolumn{5}{|l|}{ Histological grade } \\
\hline $\mathrm{H} 1-\mathrm{H} 3$ & 78 & & & \\
\hline $\mathrm{H} 4-\mathrm{H} 5$ & 18 & 0.614 & $0.341-1.107$ & 0.105 \\
\hline \multicolumn{5}{|l|}{ DDX54 expression } \\
\hline Low & 47 & & & \\
\hline High & 49 & 3.128 & $1.634-5.988$ & $0.001^{\star \star}$ \\
\hline
\end{tabular}

Statistically significant $\left({ }^{*} P<0.05\right){ }^{* *} P<0.01,{ }^{* * *} P<0.001$.
TABLE 3 | Multivariate Cox regression analysis of potential prognostic factors for colorectal cancer patients.

\begin{tabular}{lccc}
\hline Characteristics & HR & $\mathbf{9 5 \%} \mathbf{C l}$ & $\mathbf{P}$ \\
\hline Tumor size, cm & 0.515 & $0.266-0.998$ & 0.049 \\
T stage & 1.714 & $0.714-4.112$ & 0.228 \\
Lymph node metastasis & 1.750 & $0.326-9.403$ & 0.514 \\
Distant metastasis & 13.737 & $3.456-54.6$ & $<0.001^{\star \star \star}$ \\
AJCC stage & 0.742 & $0.11-5.031$ & 0.760 \\
Histological grade & 0.696 & $0.367-1.321$ & 0.268 \\
DDX54 expression & 2.485 & $1.250-4.943$ & $0.009^{\star \star}$ \\
\hline
\end{tabular}

${ }^{* *} P<0.01,{ }^{* * *} P<0.001$

cell cycle was also detected, and the results showed that the cells with DDX54 knockdown were subjected to be arrested at of G1 stage (Figure 4G). In contrary, CRC cells with enhanced DDX54 were not subjected to be arrested at G1 stage (Figures $\mathbf{4 H}, \mathbf{I}$ ).

\section{Overexpressed DDX54 Activated the P65 and AKT Pathway}

We further examined the effects of altered DDX54 on the activities of the pathways in CRC cells. We found that only the p65 and AKT was phosphorylation level obviously elevated while the others were the same as the controls in Caco2, RKO HCT116 and SW620 cells (Figure 5A). And reversely, knockdown DDX54 by RNAi conferred the reduction of phosphorylation level of p65 and AKT in these CRC cells (Figure 5B). Furthermore, the same role of DDX54 knockdown on the
A

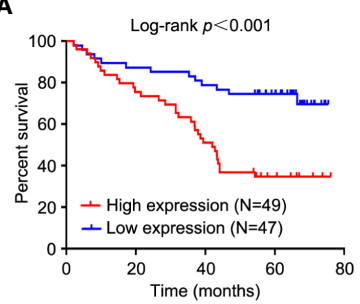

D

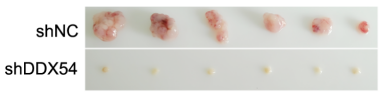

B

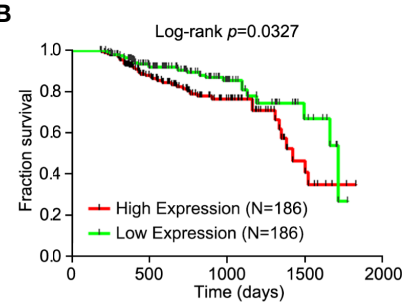

E

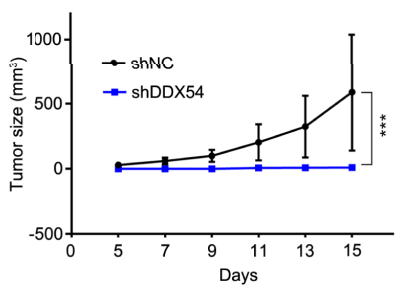

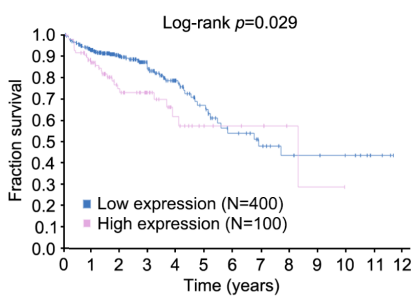

F

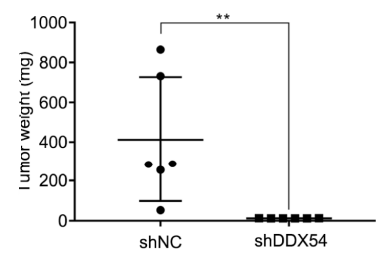

FIGURE 3 | DDX54 expression and significance in colorectal cancer was analyzed in TCGA database. (A) The relation between DDX54 mRNA level and CRC survival time was analyzed of tissue microarray. (B) Kaplan-Meier plots of the overall survival of patients stratified by DDX54 expression were analyzed on CRC patients from TCGA database with the less than 5-followup period. (C) The relation between DDX54 mRNA level and survival was analyzed on CRC patients from the Human Protein Atlas. FPKM, Fragments Per Kilobase per Million. (D) The representative images for subcutaneous tumors of DDX54 knockdown mice and relative controls. (E) The size of subcutaneous tumors was calculated at different times, ${ }^{\star \star \star} \mathrm{P}<0.001$. (F) The tumor weights of mice with control or DDX54 knockdown injection were counted, ${ }^{\star \star} \mathrm{P}<0.01$. 

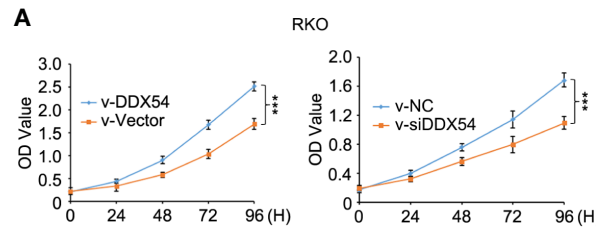

D
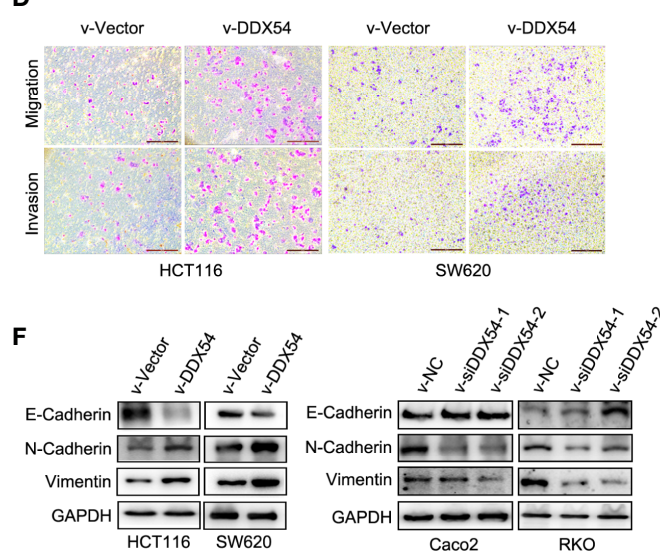

H
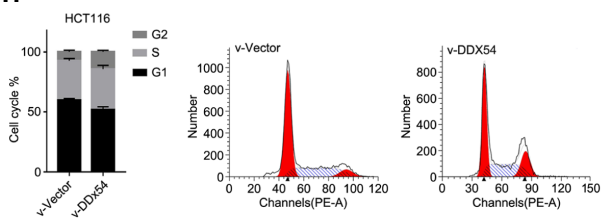
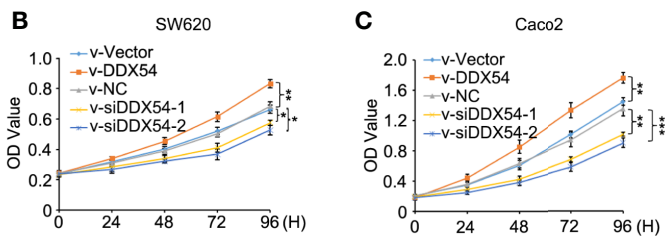

E

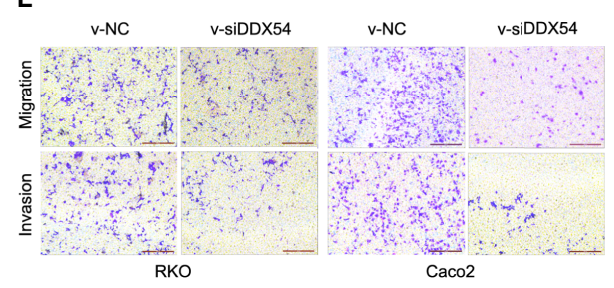

G

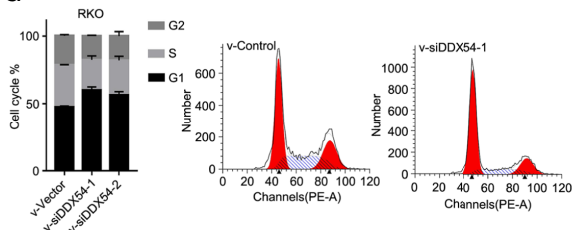

I
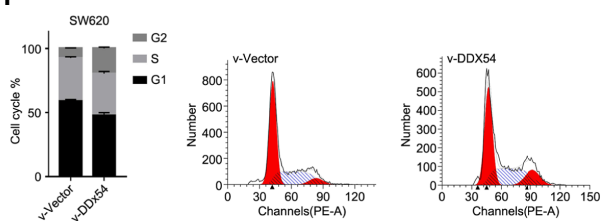

FIGURE 4 | DDX54 had a role on CRC cells. (A) The proliferative effects of DDX54 were examined through CCK8 assay in RKO stable cells, ${ }^{\star \star \star} \mathrm{P}<0.001$. (B) The role of DDX54 on proliferation was detected in SW620 cells, ${ }^{\star} P<0.05$, ${ }^{*} \mathrm{P}<0.01$. (C) DDX54 promoted the proliferation in Caco2 cells, ${ }^{\star \star} P<0.01$, ${ }^{\star \star \star} P<<0.001$. (D) The migration and invasion ability of HCT116 and SW620 was promoted by DDX54. (E) DDX54 knockdown attenuated the metastatic ability of RKO and Caco2. (F) EMT markers were determined by WB in CRC cells with DDX54 overexpression or knockdown. (G-I) DDX54 promoted the cell cycle process in CRC cells.

repressing p-p65 and p-AKT was also observed in nude mice model through immunochemical staining (Figure 5C). Meanwhile, Ki67 staining was also detected and showed that DDX54 knockdown inhibited the CRC cell proliferation which was consistent with the Cyclin D1 status regulated by DDX54 (Figures 5A, B). In CRC patients, high expressed DDX54 always showed high phosphorylation level of p65 and AKT (Figure 5D). Taken together, DDX54 regulated the cell proliferation and metastasis through activating $\mathrm{P} 65$ and AKT pathway.

To examine whether DDX54 promoted CRC progression through p65 and AKT pathway, the CRC cells were treated with Bortezomib (Btz, p-p65 inhibitors) and MK-2206 (AKT inhibitors) respectively. The results showed that BTZ and MK2206 could inhibit the cell growth respectively and neutralized the promotive effect of DDX54 on cell growth in RKO, Caco2 and HCT116 cells (Figures 6A-C). Moreover, the inhibitors could repressed the phosphorylation level of P65 and AKT, respectively, and this inhibitory effect was attenuated by enhanced DDX54 (Figures 6D, E). In addition, the metastatic ability contributed by DDX54 was also inhibited by the inhibitors of P65 and AKT pathways (Figure 6F). The EMT markers were also detected in condition of BTZ and MK2206 treatment. The results showed that E-cadherin was up-regulated by BTZ and MK-2206-treated cells with enhanced DDX54 while N-cadherin and Vimentin was down-regulated respectively compared to only DDX54 transfected cells (Figures 6G, H).

\section{DISCUSSION}

In recent years, much attention has been paid to how to improve the diagnosis and prognosis of CRC, although the great improvements have been made, the 5-year OS rate of CRC patient remains still low. Benefits from the genome sequencing, the attentions had been turned back to how to predict the cancer and the benefits from the targeted therapy. The gene functions involved in etiology of CRC had been widely investigated. In current study, we aimed to find the biomarker gene to distinguish the tumor from normal tissues. We performed the proteomic analysis in CRC patients by LC-MS, and we found 573 different proteins. Of which, DDX54 is confirmed to be highly expressed in CRC tumor tissues (Figure 2), which implied a 
A

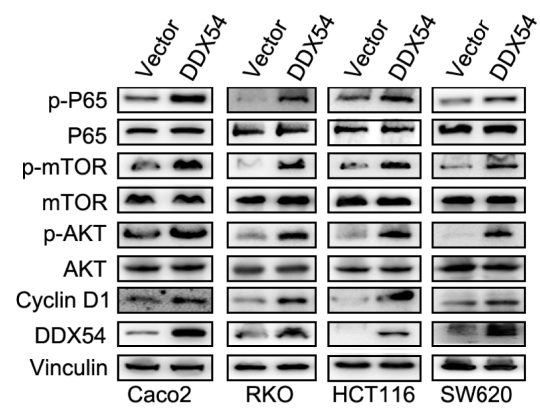

C

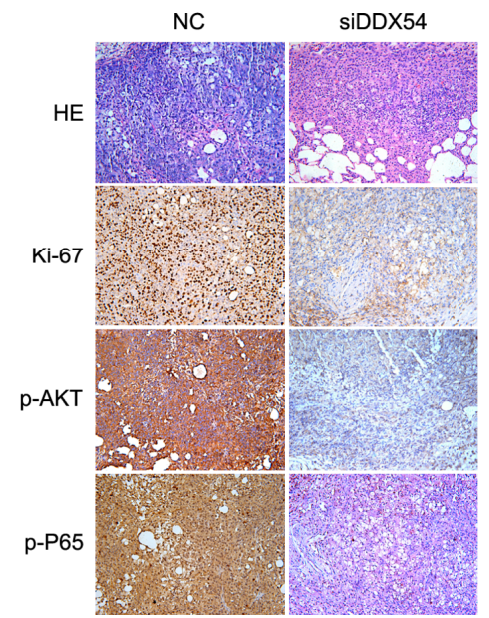

B

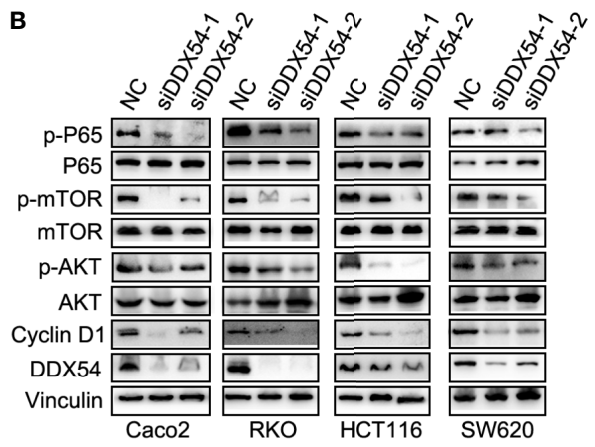

D

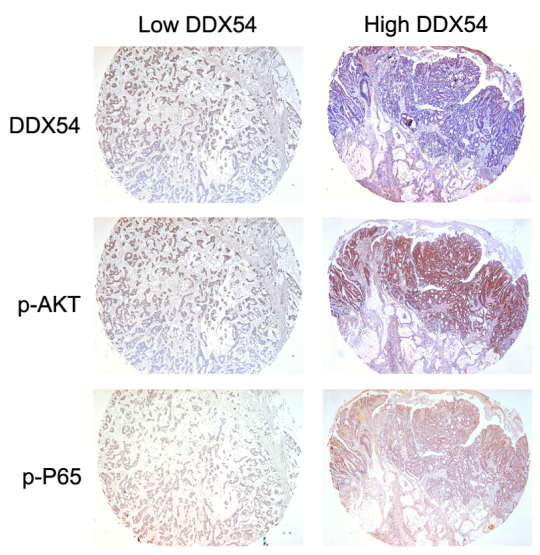

FIGURE 5 | DDX54 activated the NF-kb and AKT/mTOR pathway. (A) The enhanced DDX54 up-regulated the phosphorylation level of P65 and AKT in RKO, Caco2, SW620, and HCT116 cells. (B) The phosphorylation level of P65 and AKT was reduced by DDX54 inhibition with RNAi in RKO, Caco2, SW620, and HCT116 cells. (C) The Ki-67, p-P65, and p-AKT were examined in mice subcutaneous tumors with DDX54 knockdown. (D) The level of p-P65 and p-AKT in CRC patients with high DDX54 was higher than those with low DDX54.

closely association of DDX54 with colon cancers. Recently, increasing publications about DDX RNA helicase family members have been described an important role on CRC development. DDX5 can bind to Lnc-NEAT1 to promote its stability and this complex can sequentially activated Wnt signaling (30). In another research, the authors found p68, also named as DDX5, and $\beta$-catenin increased RelA mRNA and protein expression. DDX5 was perceived to associate with RelA promoter while $\beta$-catenin at the TCF4/LEF (TBE) sites thereby potentiating RelA transcription (31). DDX27 was also observed to be highly expressed in CRC tissues and increased the proliferation and motility through promote p65 to bind to the promoter region of its target genes (10). On the other hand, highly expressed DDX27 was found to promote promotes sensitivity to 5-fluorouracil (32). Moreover, DDX3 and DDX1 were also found to have an oncogenic role on CRC $(33,34)$.

Furthermore, to investigate the underlying mechanism for DDX54 to promote CRC progression, we showed that DDX54 had a promotive effect on CRC cell proliferation and metastasis through activating NF- $\mathrm{kb}$ and AKT-mTOR signaling pathway
(Figure 5). When we blocked the NF- $\kappa b$ and AKT-mTOR signaling pathway with BTZ and MK-2206, the cell growth and mobility were both inhibited. In DDX54 overexpressed cells, the effect on proliferation and metastasis of inhibitors was reversed to the primary level (Figure 6). Here, we found that the concurrent activation of NF- Kb and AKT pathway was necessary for the role of DDX54 in CRC. The NF- $\mathrm{kb}$ and AKT pathway were both activated in some certain cancers due to unknown mechanism by which the cancer cells had some selective benefits $(35,36)$. In worth to date that inhibiting either pathway alone had partial effects on tumor proliferation and metastasis (37). One reasonable explanation is that these two pathways activate a same set of downstream targets. If so, inhibition of either alone would be insufficient to block these targets.

In the current assay, we also indicated the association between the high expressed level DDX54 and the prognosis of CRC patients. By tissue microarray, we found that the CRC patients with overexpressed DDX54 always had the poor survival time compared to those with lower DDX54. Since DDX54 belong to the RNA helicase family, regulated a 
A

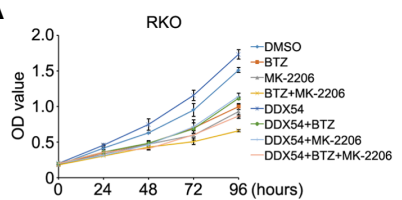

B

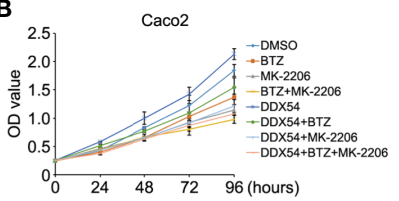

C

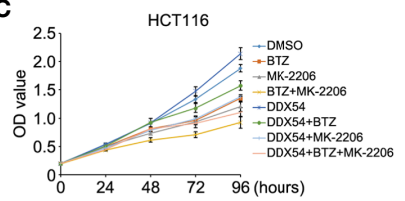

D
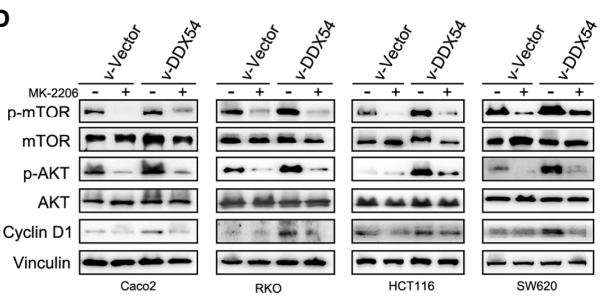

E
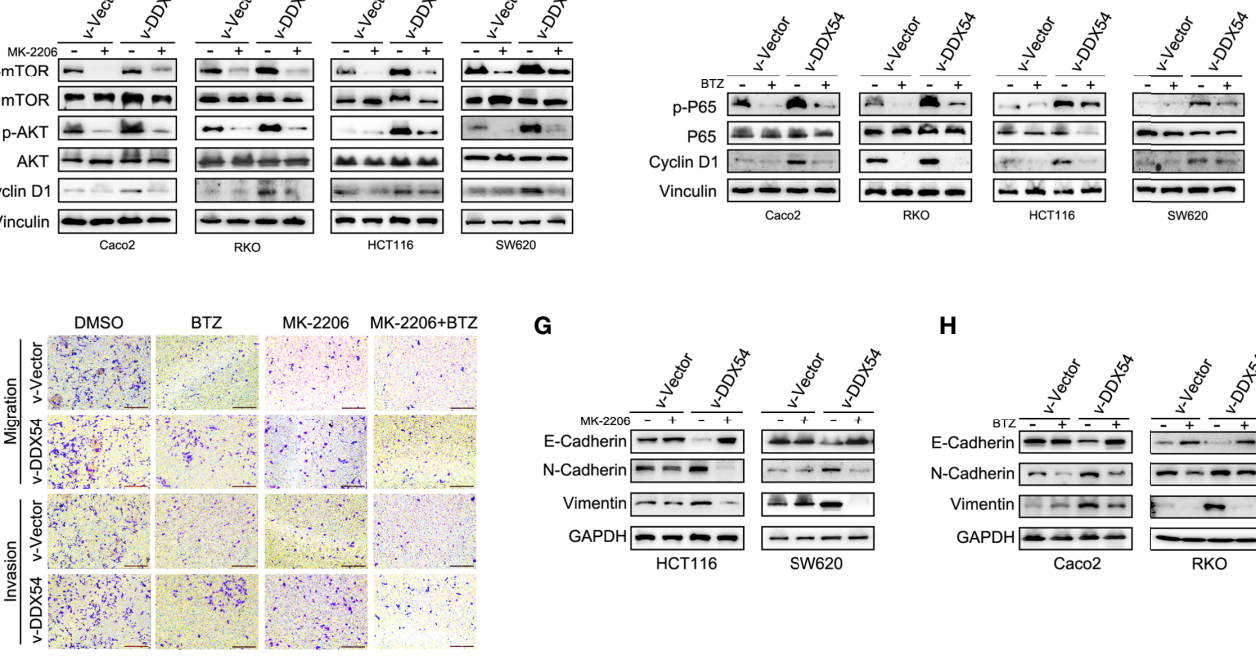

G

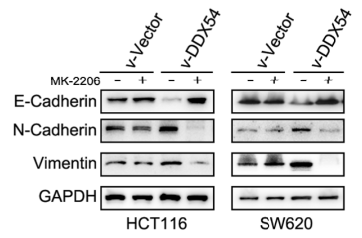

H

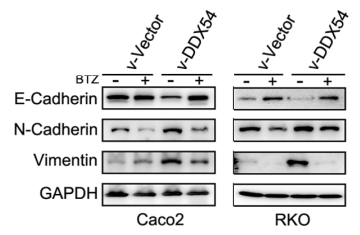

FIGURE 6 | Inhibition of NF-kb and AKT/mTOR pathway weakened the carcinogenic effects of CRC cells by DDX54. (A-C) BTZ (Bortezomib, 2 nM) and MK2206 $(1 \mu \mathrm{M})$ (inhibitor of NF-kb and AKT pathway respectively, Selleck) repressed the proliferative role of DDX54 on CRC cells. (D, E) The phosphorylation level of P65 and AKT was determined in CRC cells with different treatments of DDX54 and inhibitors. (F) The metastasis ability was weakened by inhibitors. (G, H) EMT markers were detected in CRC cells with DDX54 and inhibitor treatments.

set of genes which participated in the important cell process. In summary, we found the high level DDX54 was associated with the tumorigenesis. DDX54 could promote the proliferation and motility through elevating the p-p65 and p-AKT level.

\section{DATA AVAILABILITY STATEMENT}

The data sets presented in this study can be found in online repositories. The names of the repository/repositories and accession number(s) can be found in the article/Supplementary Material.

\section{ETHICS STATEMENT}

The studies involving human participants were reviewed and approved by the ethics committees of Shanghai Jiao Tong University School of Medicine. The patients/participants provided their written informed consent to participate in this study. The animal study was reviewed and approved by the ethics committees of Shanghai Jiao Tong University School of Medicine. The animal study was reviewed and approved by the ethics committees of Shanghai Jiao Tong University School of Medicine.

\section{AUTHOR CONTRIBUTIONS}

L-DZ, G-HF, and YY conceived and designed the research. YY, J-LW, L-LM performed the experiments and analyzed the data, C-TH, X-QS contributed to collect clinical samples, Z-PH, Z-WY conducted the IHC. All authors have made a substantial, direct, and intellectual contribution to the work and approved it for publication.

\section{FUNDING}

This work was supported in part by First Round of 3-year Action Plan to promote clinical skills and clinical innovation in Municipal Hospitals of Shanghai (16CR2039B), Shanghai Municipal Key Clinical Specialty Construction Project (NOshslczdzk01303).

\section{SUPPLEMENTARY MATERIAL}

The Supplementary Material for this article can be found online at: https://www.frontiersin.org/articles/10.3389/fonc.2021.650360/ full\#supplementary-material 


\section{REFERENCES}

1. Brenner H, Kloor M, Pox CP. Colorectal cancer. Lancet (2014) 383:1490-502. doi: 10.1016/S0140-6736(13)61649-9

2. Board RE0, Valle JW. Metastatic colorectal cancer: current systemic treatment options. Drugs (2007) 67:1851-67. doi: 10.2165/00003495-200767130-00004

3. Ferlay J, Autier P, Boniol M, Heanue M, Colombet M, Boyle P. Estimates of the cancer incidence and mortality in Europe in 2006. Ann Oncol (2007) 18:581-92. doi: 10.1093/annonc/mdl498

4. Pancione M, Remo A, Colantuoni V. Genetic and epigenetic events generate multiple pathways in colorectal cancer progression. Pathol Res Int (2012) 509348:2012. doi: 10.1155/2012/509348

5. Linder P, Jankowsky E. From unwinding to clamping - the DEAD box RNA helicase family. Nat Rev Mol Cell Biol (2011) 12:505-16. doi: 10.1038/nrm3154

6. Hashemi V, Masjedi A, Hazhir-Karzar B, Tanomand A, Shotorbani SS, Hojjat-Farsangi M, et al. The role of DEAD-box RNA helicase p68 (DDX5) in the development and treatment of breast cancer. J Cell Physiol (2019) 234:5478-87. doi: 10.1002/jcp.26912

7. Wang Z, Luo Z, Zhou L, Li X, Jiang T, Fu E. DDX5 promotes proliferation and tumorigenesis of non-small-cell lung cancer cells by activating beta-catenin signaling pathway. Cancer Sci (2015) 106:1303-12. doi: 10.1111/cas.12755

8. Xie M, Vesuna F, Tantravedi S, Bol GM, Voss MR, Nugent K, et al. RK-33 Radiosensitizes Prostate Cancer Cells by Blocking the RNA Helicase DDX3. Cancer Res (2016) 76:6340-50. doi: 10.1158/0008-5472.CAN-16-0440

9. Chang PC, Chi CW, Chau GY, Li FY, Tsai YH, Wu JC, et al. DDX3, a DEAD box RNA helicase, is deregulated in hepatitis virus-associated hepatocellular carcinoma and is involved in cell growth control. Oncogene (2006) 25:19912003. doi: 10.1038/sj.onc.1209239

10. Tang J, Chen H, Wong CC, Liu D, Li T, Wang X, et al. DEAD-box helicase 27 promotes colorectal cancer growth and metastasis and predicts poor survival in CRC patients. Oncogene (2018) 37:3006-21. doi: 10.1038/s41388-018-0196-1

11. Zhou J, Yong WP, Yap CS, Vijayaraghavan A, Sinha RA, Singh BK, et al. An integrative approach identified genes associated with drug response in gastric cancer. Carcinogenesis (2015) 4(4):441-451. doi: 10.1093/carcin/bgv014

12. Rajendran RR, Nye AC, Frasor J, Balsara RD, Martini PG, Katzenellenbogen BS. Regulation of nuclear receptor transcriptional activity by a novel DEAD box RNA helicase (DP97). J Biol Chem (2003) 278:4628-38. doi: 10.1074/jbc.M210066200

13. Kanno Y, Serikawa T, Inajima J, Inouye Y. DP97, a DEAD box DNA/RNA helicase, is a target gene-selective co-regulator of the constitutive androstane receptor. Biochem Biophys Res Commun (2012) 426:38-42. doi: 10.1016/j.bbrc.2012.08.027

14. Milek M, Imami K, Mukherjee N, Bortoli FD, Zinnall U, Hazapis O, et al. DDX54 regulates transcriptome dynamics during DNA damage response. Genome Res (2017) 27:1344-59. doi: 10.1101/gr.218438.116

15. Tokunaga H, Seiwa C, Yoshioka N, Mizoguchi K, Yamamoto M, Asou H, et al. An Extract of Chinpi, the Dried Peel of the Citrus Fruit Unshiu, Enhances Axonal Remyelination via Promoting the Proliferation of Oligodendrocyte Progenitor Cells. Evidence-Based Complement Altern Med (2016) 2016:8692698. doi: 10.1155/2016/8692698

16. Zhang Y, Guo H, Zhang H. SNHG10/DDX54/PBX3 Feedback Loop Contributes to Gastric Cancer Cell Growth. Dig Dis Sci (2020). doi: 10.1007/s10620-020-06488-9

17. Perkins ND. The diverse and complex roles of NF-kappaB subunits in cancer. Nat Rev Cancer (2012) 12:121-32. doi: 10.1038/nrc3204

18. Fan Y, Mao R, Yang J. NF-kappaB and STAT3 signaling pathways collaboratively link inflammation to cancer. Protein Cell (2013) 4:176-85. doi: 10.1007/s13238-013-2084-3

19. Timucin AC, Basaga H. Pro-apoptotic effects of lipid oxidation products: HNE at the crossroads of NF-kappaB pathway and anti-apoptotic Bcl-2. Free Radical Biol Med (2017) 111:209-18. doi: 10.1016/j.freeradbiomed.2016.11.010

20. Prasad S, Tyagi AK. Ginger and its constituents: role in prevention and treatment of gastrointestinal cancer. Gastroenterol Res Pract (2015) 2015:142979. doi: 10.1155/2015/142979

21. McCubrey JA, Steelman LS, Chappell WH, Abrams SL, Wong EW, Chang F, et al. Roles of the Raf/MEK/ERK pathway in cell growth, malignant transformation and drug resistance. Biochim Biophys Acta (2007) 1773:1263-84. doi: 10.1016/ j.bbamcr.2006.10.001
22. Shaw RJ, Cantley LC. Ras, PI(3)K and mTOR signalling controls tumour cell growth. Nature (2006) 441:424-30. doi: 10.1038/nature04869

23. Samuels Y, Wang Z, Bardelli A, Silliman N, Ptak J, Szabo S, et al. High frequency of mutations of the PIK3CA gene in human cancers. Science (2004) 304:554. doi: 10.1126/science.1096502

24. Vivanco I, Sawyers CL. The phosphatidylinositol 3-Kinase AKT pathway in human cancer. Nat Rev Cancer (2002) 2:489-501. doi: 10.1038/nrc839

25. Tyanova S, Temu T, Cox J. The MaxQuant computational platform for mass spectrometry-based shotgun proteomics. Nat Protoc (2016) 11:2301-19. doi: 10.1038/nprot.2016.136

26. Chen G, Chen J, Liu H, Chen S, Zhang Y, Li P, et al. Comprehensive Identification and Characterization of Human Secretome Based on Integrative Proteomic and Transcriptomic Data. Front Cell Dev Biol (2019) 7:299. doi: 10.3389/fcell.2019.00299

27. Cox J, Hein MY, Luber CA, Paron I, Nagaraj N, Mann M. Accurate proteomewide label-free quantification by delayed normalization and maximal peptide ratio extraction, termed MaxLFQ. Mol Cell Proteomics (2014) 13:2513-26. doi: 10.1074/mcp.M113.031591

28. Brenna S, Altmeppen HC, Mohammadi B, Rissiek B, Schlink F, Ludewig P, et al. Characterization of brain-derived extracellular vesicles reveals changes in cellular origin after stroke and enrichment of the prion protein with a potential role in cellular uptake. J Extracell Vesicles (2020) 9:1809065. doi: 10.1080/20013078.2020.1809065

29. Zu L, Xue Y, Wang J, Fu Y, Wang X, Xiao G, et al. The feedback loop between miR-124 and TGF-beta pathway plays a significant role in non-small cell lung cancer metastasis. Carcinogenesis (2016) 37:333-43. doi: 10.1093/carcin/ bgw011

30. Zhang M, Weng W, Zhang Q, Wu Y, Ni S, Tan C, et al. The lncRNA NEAT1 activates $\mathrm{Wnt} /$ beta-catenin signaling and promotes colorectal cancer progression via interacting with DDX5. J Hematol Oncol (2018) 11:113. doi: 10.1186/s13045-018-0656-7

31. Khare V, Tabassum S, Chatterjee U, Chatterjee S, Ghosh MK. RNA helicase p68 deploys beta-catenin in regulating RelA/p65 gene expression: implications in colon cancer. J Exp Clin Cancer Res (2019) 38:330. doi: 10.1186/s13046019-1304-y

32. Yang C, Li D, Bai Y, Song S, Yan P, Wu R, et al. DEAD-box helicase 27 plays a tumor-promoter role by regulating the stem cell-like activity of human colorectal cancer cells. Onco Targets Ther (2019) 12:233-41. doi: 10.2147/ OTT.S190814

33. He TY, Wu DW, Lin PL, Wang L, Huang CC, Chou MC, et al. DDX3 promotes tumor invasion in colorectal cancer via the CK1epsilon/Dvl2 axis. Sci Rep (2016) 6:21483. doi: 10.1038/srep21483

34. Tanaka K, Ikeda N, Miyashita K, Nuriya H, Hara T. DEAD box protein DDX1 promotes colorectal tumorigenesis through transcriptional activation of the LGR5 gene. Cancer Sci (2018) 109:2479-89. doi: 10.1111/cas.13661

35. Dariya B, Nagaraju GP. Advanced glycation end products in diabetes, cancer and phytochemical therapy. Drug Discov Today (2020) 25:1614-23. doi: 10.1016/j.drudis.2020.07.003

36. Zhang Z, Yang L, Hou J, Tian S, Liu Y. Molecular mechanisms underlying the anticancer activities of licorice flavonoids. J Ethnopharmacol (2020) 267:113635. doi: 10.1016/j.jep.2020.113635

37. Hoeflich A, Bielohuby M. Mechanisms of adrenal gland growth: signal integration by extracellular signal regulated kinases1/2. J Mol Endocrinol (2009) 42:191-203. doi: 10.1677/JME-08-0160

Conflict of Interest: The authors declare that the research was conducted in the absence of any commercial or financial relationships that could be construed as a potential conflict of interest.

Copyright (C) 2021 Yu, Wang, Meng, Hu, Yan, He, Shi, Fu and Zu. This is an openaccess article distributed under the terms of the Creative Commons Attribution License (CC BY). The use, distribution or reproduction in other forums is permitted, provided the original author(s) and the copyright owner(s) are credited and that the original publication in this journal is cited, in accordance with accepted academic practice. No use, distribution or reproduction is permitted which does not comply with these terms. 